\title{
Association of furosemide or hydrochlorothiazide use with risk of atrial fibrillation post pacemaker implantation among elderly patients
}

\author{
Da-Wei Lin ${ }^{1 \#}$, Feng Jiang ${ }^{2 \#} \wedge$, Chen Wu ${ }^{1}$, Yi-Gang Li $^{1}$, Xi Zhang ${ }^{3}$, Yao-Sheng Wang ${ }^{1,3} \wedge$ \\ ${ }^{1}$ Department of Cardiology, Xinhua Hospital Affiliated to Shanghai Jiao Tong University School of Medicine, Shanghai, China; ${ }^{2}$ Chongming Branch, \\ Clinical Research \& Innovation Unit, Xinhua Hospital Affiliated to Shanghai Jiao Tong University School of Medicine, Shanghai, China; ${ }^{3}$ Clinical \\ Research \& Innovation Unit, Xinhua Hospital of Shanghai Jiao Tong University School of Medicine, Shanghai, China \\ Contributions: (I) Conception and design: YS Wang; (II) Administrative support: None; (III) Provision of study materials or patients: None; (IV) \\ Collection and assembly of data: All authors; (V) Data analysis and interpretation: All authors; (VI) Manuscript writing: All authors; (VII) Final \\ approval of manuscript: All authors. \\ \#These authors contributed equally to this work. \\ Correspondence to: Yao-Sheng Wang. Department of Cardiology, Xinhua Hospital Affiliated to Shanghai Jiao Tong University School of Medicine, \\ Shanghai 200092, China. Email: wangyaosheng@xinhuamed.com.cn.
}

Background: Atrial fibrillation (AF) induced by artificial pacing is directly related to atrial remodeling. Previous basic research has shown that furosemide aggravates pathologic myocardial remodeling while hydrochlorothiazide alleviates it. However, whether furosemide or hydrochlorothiazide plays a role in developing AF after pacemaker implantation remains unknown. The study aims to investigate the association between oral furosemide or hydrochlorothiazide and the risk of developing AF after pacemaker implantation. Methods: After a review of electronic medical records, elderly patients with pacemaker implantation and without a known baseline history of AF were included and information on their use of daily oral furosemide or hydrochlorothiazide was extracted. New incident AF cases were confirmed via the records of outpatient visits. A Cox proportional-hazards model was used to evaluate the association between daily oral furosemide or hydrochlorothiazide and risk of developing AF after pacemaker implantation, after adjustment for potential confounders.

Results: Among a total of 551 patients aged more than 65 years, 157 AF cases were identified after

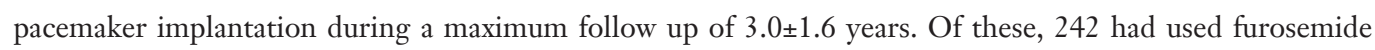
and 97 had used hydrochlorothiazide therapy. Patients taking daily oral furosemide had a relatively higher risk of $\mathrm{AF}$ after pacemaker implantation [hazard ratio (HR): 1.507, 95\% confidence interval (CI): 1.0362.192; $\mathrm{P}=0.032$ ] after being adjusted for related disease and prescribed medications, while oral taking of hydrochlorothiazide was shown to be a non-effective factor (HR: 0.666, 95\% CI: 0.413-1.074), which had no statistical significance.

Conclusions: Daily oral furosemide might increase the risk of developing AF after pacemaker implantation in elderly patients, while hydrochlorothiazide has no detrimental effect.

Keywords: Furosemide; hydrochlorothiazide; atrial fibrillation (AF); pacemaker implantation; elderly patients

Submitted Mar 13, 2021. Accepted for publication May 17, 2021.

doi: 10.21037/atm-21-1792

View this article at: http://dx.doi.org/10.21037/atm-21-1792

^ ORCID: Feng Jiang, 0000-0002-8071-8279; Yao-Sheng Wang, 0000-0001-5109-1473. 


\section{Introduction}

Pacemaker implantation is currently a common and effective method to treat many types of bradycardia arrhythmias $(1,2)$. However, a significant amount of research including the MOST study and CTOPP study has shown that the incidence of atrial fibrillation (AF) in pacemaker implanted populations increases from $3 \%$ to $15-30 \%$ (3-5). AF induced by artificial pacing is widely considered to be directly related to atrial pathologic remodeling, which includes structural pathologic remodeling and electrical remodeling. In addition, sympathetic activation, inflammation, and pacing mode are associated with the risk of developing $\mathrm{AF}$ after pacemaker implantation $(6,7)$. While much attention has recently been paid to the effects of commonly used cardiovascular drugs, such as ACEI, ARB, and statins in the occurrence of $\mathrm{AF}$ in patients implanted with a permanent pacemaker, few studies have focused on the effect of diuretics $(8,9)$.

In keeping with clinical guidelines, a significant proportion of elderly patients are taking diuretics daily to regulate blood pressure, reduce the burden on the heart, and eliminate edema to counter the impacts caused by hypertension (HTN), heart failure (HF), abnormal renal and liver function, and other diseases $(10,11)$. Furthermore, elderly individuals are prone to undergo pacemaker implantation for bradycardia arrhythmias resulting in a higher risk of $\mathrm{AF}$ (12). Therefore, the potential association between diuretics use and the occurrence of AF after pacemaker implantation in the elderly deserves further investigation.

Thiazides, loop diuretics, and potassium-sparing diuretics make up the main composition of diuretics, among which furosemide and hydrochlorothiazide are the most used. By removing excess liquid from the body, daily oral furosemide and hydrochlorothiazide can help reduce blood pressure, decrease the burden on heart, and alleviate chronic HF. However, previous basic research has suggested that these medications may play different roles in the development of $\mathrm{AF}$ (13). Furosemide enhances plasma renin, angiotensin II (Ang II) and aldosterone, activates sympathetic nerves, and reduces the level of serum magnesium, promoting the development of AF (14). Conversely, hydrochlorothiazide reduces the serum level of Ang II, thereby inhibiting the transforming growth factor (TGF)- $\beta /$ Smads signaling pathway to alleviate heart structural pathologic remodeling (15). These mechanisms may be strongly associated with the development of $\mathrm{AF}$ after pacemaker implantation in the elderly. Therefore, it is of great significance to explore the effects of diuretics on the development of AF after pacemaker implantation in the clinically real world and gain evidence for diuretic selection in elderly pacing patients. This study aims to evaluate the association of furosemide or hydrochlorothiazide use with the risk of $\mathrm{AF}$ after pacemaker implantation among elderly patients. We present the following article in accordance with the STROBE reporting checklist (available at http:// dx.doi.org/10.21037/atm-21-1792).

\section{Methods}

A single-center, retrospective, observational study was conducted to assess the association of furosemide or hydrochlorothiazide use with the subsequent risk of developing AF in elderly patients who were implanted with pacemakers.

\section{Study subject}

After the de-identification of personal information, patients aged more than 65 years and without a known baseline history of AF who received pacemaker implantation in the Xinhua Hospital Affiliated to Shanghai Jiao Tong University School of Medicine from 2012 to 2018 were included based on a review of their electronical medical records (EMR), and information on their daily use of oral furosemide or hydrochlorothiazide was extracted. The exclusion criteria for the study were any of the following: (I) severe liver and renal dysfunction, (II) abnormal thyroid function (hyperthyroidism), (III) congenital heart disease, (IV) past history of heart surgery history, (V) severe valvular heart disease, (VI) patients diagnosed with AF before the index date, either paroxysmal or permanent. Patients who were diagnosed with $\mathrm{AF}$ within 3 months after pacemaker implantation were also excluded, since it is widely accepted that at least 3 months are needed for cardiac remodeling to induce $\mathrm{AF}$ and this protocol has also been adopted in (16). Ethical approval was granted by the Medical Ethics Committee of the Xinhua Hospital Chongming Branch (CMEC-2020-KT-14) and obeyed the principles of the Declaration of Helsinki (as revised in 2013). Besides, the study was waived the need for informed consent due to the observational character of the study.

\section{Medication information collection}

The main exposure of interest was daily oral furosemide 
or hydrochlorothiazide, which could be identified from prescription records.

\section{Primary outcomes}

AF cases were defined by checking the EMR for evidence of a diagnosis made by a professional physician at any ambulatory visit, at discharge, or during post-operation follow-up based on the International Classification of Diseases, 10th Revision, diagnosis code (ICD-10: I48).

\section{Covariates}

A retrospective review using EMR and database information was conducted for evidence of HTN, diabetes mellitus (DM), or HF. The left ventricular ejection fraction (LVEF) and NYHA Functional Classification of each patient was then determined, and as these factors are commonly accepted as closely related to the development of AF, they were defined as covariates. Data on other prescribed related medications including ACEI/ARB, $\beta$-blockers, calcium-channel blockers (CCBs), statins, diuretics except furosemide (DEF), and diuretics except hydrochlorothiazide (DEH) were also identified.

\section{Statistical analysis}

Continuous variables were expressed as mean values $\pm S D$, whereas categorical variables were expressed as frequencies between subjects with and without taking furosemide or hydrochlorothiazide. Associations between categorical variables were tested by Pearson's $\chi^{2}$ test and KolmogorovSmirnov test was used to examine whether the data were normally distributed. Comparisons for the continuous data showing normal distribution were performed using the Student's $t$-test or otherwise by the Mann-Whitney $\mathrm{U}$ test. Cox proportional models were established to calculate the hazard ratio (HR) and $95 \%$ confidence interval (CI) for evaluating the association between oral furosemide or hydrochlorothiazide and $\mathrm{AF}$ after pacemaker implantation with adjustment for potential confounders, including age, gender, documented comorbidities, and concomitant medications. Kaplan-Meier survival analysis was used to indicate the AF-free survival in furosemide and hydrochlorothiazide groups. Statistical processes were completed by STATA 15.1 software.

\section{Results}

\section{Patient characteristics}

A total of 551 patients aged more than 65 years who met the study inclusion criteria were included for analyses and $157 \mathrm{AF}$ cases were identified after pacemaker implantation during a follow up of $3.0 \pm 1.6$ years. Among all the enrolled individuals, $309(56.1 \%)$ subjects received furosemide therapy while 97 (17.6\%) took hydrochlorothiazide. The baseline characteristics of all subjects are summarized in Tables 1 and 2. The rate of occurrence of AF after pacemaker implantation in those who received furosemide was significantly higher than in those without it $37.2 \%$ vs. $21.7 \%, \mathrm{P}<0.001)$, and they were markedly older $(83.8 \pm 5.9$ vs. $81.4 \pm 7.5, \mathrm{P}<0.001)$. In addition, compared with those not taking it, patients prescribed with furosemide were more likely to receive cardioprotective medications including ACEI (43.0\% vs. 34.0\%, $\mathrm{P}=0.031)$ and other diuretics except furosemide ( $94.6 \%$ vs. $55.3 \%, \mathrm{P}<0.001)$. The furosemide group also had a higher rate of HTN (77.3\% vs. $66.9 \%, \mathrm{P}=0.029)$, $\mathrm{HF}(63.6 \%$ vs. $27.8, \mathrm{P}<0.001)$, and diabetes $(28.5 \%$ vs. $19.4 \%, \mathrm{P}=0.012)$ (Table 1). Subjects who received hydrochlorothiazide therapy were more likely to use ACEI (52.6\% vs. 34.8\%, $\mathrm{P}=0.001)$, ARB (87.6\% vs. $55.9 \%, \mathrm{P}<0.001)$, CCB (79.4\% vs. 59.0\%, $\mathrm{P}<0.001)$, and statins $(73.2 \%$ vs. $57.7 \%, \mathrm{P}=0.005)$, and had a higher risk of developing HTN (88.7\% vs. 69.2\%, $\mathrm{P}<0.001)$ than those not taking daily oral hydrochlorothiazide (Table 2).

\section{Outcome: AF after pacemaker implantation}

The median duration of follow-up of the entire retrospective cohort was 2.9 years. By using multivariate Cox hazard regression analysis, daily oral furosemide use demonstrated stable association with a raised risk of developing new AF after pacemaker implantation, when compared with subjects not taking it (HR: 1.507; 95\% CI: $1.036-2.192, \mathrm{P}=0.032$ ) and this remained statistically significant after adjustment for other clinical covariates including age, gender, smoking and drinking, medication use (ACEI, ARB, $\beta$-blocker, CCB, statins, DEF, DEH), and a history of chronic disease (HTN, NYHA functional class, ischemic stroke). In contrast receiving hydrochlorothiazide therapy was shown to be a non-effective factor (HR: 0.666; 95\% CI: 0.413-1.074, $\mathrm{P}=0.095)$, with no statistical significance (Table 3). 
Table 1 Characteristics of furosemide users and non-users

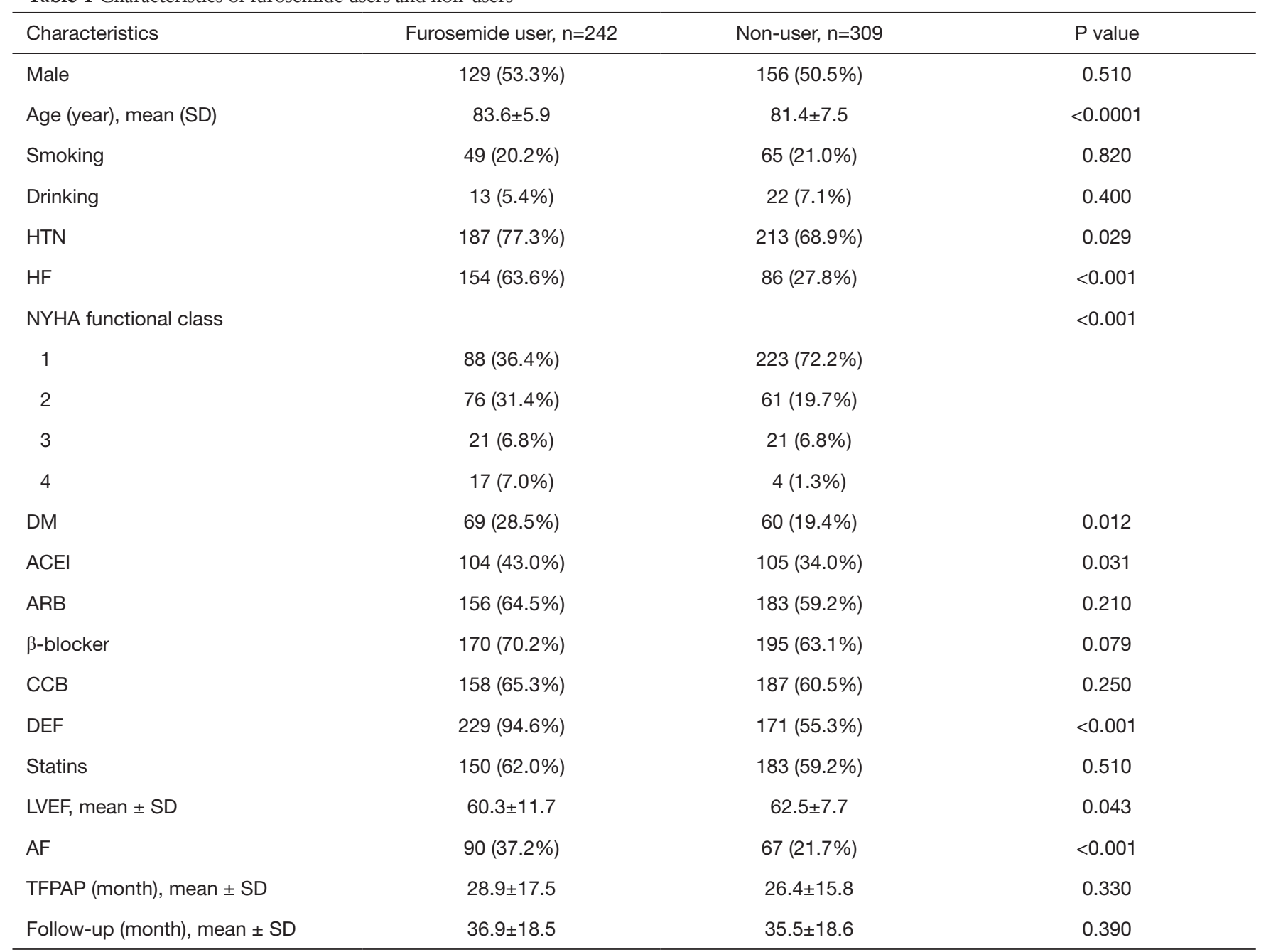

HTN, hypertension; HF, heart failure; NYHA, New York Heart Association; DM, diabetes mellitus; ACEl, angiotensin-converting enzyme inhibitor; ARB, angiotensin-receptor blocker; CCB, calcium channel blocker; DEF, diuretic except furosemide; AF, atrial fibrillation; TFPAP, time from pacemaker implantation to atrial fibrillation occurrence.

Kaplan-Meier survival analysis indicated that patients taking furosemide were also associated with worse AF-free survival after pacemaker implantation (Figure 1), while a trend of preventing the development of AF after pacemaker implantation by using hydrochlorothiazide failed to reach clinical or statistical significance $(\mathrm{P}=0.095)$ (Figure 2).

\section{Discussion}

AF induced by artificial pacing is considered directly related to atrial pathologic remodeling, which includes structural pathologic remodeling, and electrical remodeling. Sympathetic activation, inflammation, and pacing mode are also associated with the occurrence of AF after pacemaker implantation. On the other hand, some elderly patients undergo pacemaker implantation for bradycardia arrhythmias. As an independent risk factor for the development of AF, aging may inevitably lead to myocardial hypertrophy and fibrosis, sympathetic activation, and decreased renal function, which may promote the development of $\mathrm{AF}(17,18)$. Therefore, factors that influence myocardial remodeling may affect the development of $\mathrm{AF}$ in patients with a permanent pacemaker, especially in elderly individuals.

Basic research has shown that furosemide aggravates myocardial remodeling via increasing the serum level 
Table 2 Characteristics of hydrochlorothiazide users and non-users

\begin{tabular}{|c|c|c|c|}
\hline Characteristics & Hydrochlorothiazide user, $\mathrm{n}=97$ & Non-user, n=454 & $P$ value \\
\hline Age (year), mean $\pm S D$ & $82.2 \pm 7.2$ & $82.4 \pm 6.9$ & 0.840 \\
\hline Smoking & $28(28.9 \%)$ & $86(18.9 \%)$ & 0.029 \\
\hline Drinking & $11(11.3 \%)$ & $24(5.3 \%)$ & 0.026 \\
\hline $\mathrm{HF}$ & $46(47.4 \%)$ & $194(42.7 \%)$ & 0.400 \\
\hline NYHA functional class & & & 0.530 \\
\hline 1 & $51(52.6 \%)$ & $260(57.3 \%)$ & \\
\hline 2 & $27(27.8 \%)$ & $110(24.2 \%)$ & \\
\hline DM & $23(23.7 \%)$ & $106(23.3 \%)$ & 0.940 \\
\hline ACEI & $51(52.6 \%)$ & $158(34.8 \%)$ & 0.001 \\
\hline ARB & $85(87.6 \%)$ & $254(55.9 \%)$ & $<0.001$ \\
\hline$\beta$-blocker & $72(74.2 \%)$ & $293(64.5 \%)$ & 0.067 \\
\hline $\mathrm{CCB}$ & $77(79.4 \%)$ & $268(59.0 \%)$ & $<0.001$ \\
\hline DEF & $70(72.2 \%)$ & $316(69.6 \%)$ & 0.620 \\
\hline Statins & $71(73.2 \%)$ & $262(57.7 \%)$ & 0.005 \\
\hline LVEF, mean \pm SD & $60.8 \pm 8.9$ & $61.6 \pm 10.1$ & 0.570 \\
\hline
\end{tabular}

HTN, hypertension; HF, heart failure; NYHA, New York Heart Association; DM, diabetes mellitus; ACEl, angiotensin-converting enzyme inhibitor; ARB, angiotensin-receptor blocker; $\mathrm{CCB}$, calcium channel blocker; $\mathrm{DEH}$, diuretic except hydrochlorothiazide; AF, atrial fibrillation; TFPAP, time from pacemaker implantation to atrial fibrillation occurrence.

of Ang II and aldosterone (19). The activating effect of furosemide on the renin-angiotensin-aldosterone system (RAAS) is also evidenced by significantly greater serum Ang II and aldosterone, as shown in a previous study (20). Ang II is a powerful promoter of fibrosis, which increases collagen content and fibroblast proliferation, which in turn disturbs heart conduction velocity and induces regional conduction blocks, resulting in ideal conditions for the occurrence of AF. Elevated plasma and myocardial aldosterone levels are also widely reported to be highly associated with the development of cardiac fibrosis (21). Myocardium and interstitial fibrosis are marked by an increase in atrial connective tissue growth factor (CTGF) and plasma Interleukin-6 (IL-6) levels, while aldosterone induces their expression through their activation of the NF$\kappa \mathrm{B}(22)$. Thus, abnormal electrical excitability and atrial morphological structure changes induced by pacemaker implantation may lead to increased Ang II and aldosterone levels, and daily oral furosemide may exacerbate these effects. Loop diuretics, including furosemide, have been reported to trigger sympathetic nervous system activation (23) and one study demonstrated that furosemide can directly activate the renal sympathetic nervous system, independent from the RASS (24). This phenomenon may well be elicited by a change in the local micro-environment of the afferent nerve endings in the renal interstitium, which 
Table 3 Associations between furosemide or hydrochlorothiazide use and risk of atrial fibrillation after pacing among Chinese patients

\begin{tabular}{|c|c|c|c|}
\hline Variables & Case/N & \multicolumn{2}{|c|}{ Cox model } \\
\hline \multicolumn{4}{|l|}{ Furosemide } \\
\hline Use & $90 / 242$ & 1.507 (1.036, 2.192) & 0.032 \\
\hline Non-user & $67 / 309$ & $0.664(0.456,0.965)$ & 0.032 \\
\hline \multicolumn{4}{|c|}{ Hydrochlorothiazide } \\
\hline Use & $22 / 97$ & $0.666(0.413,1.074)$ & 0.095 \\
\hline Non-user & $135 / 454$ & $1.501(0.931,2.420)$ & 0.095 \\
\hline
\end{tabular}

Model adjustment: age, gender, smoking and drinking, medication use (ACEI, ARB, $\beta$ blocker, CCB, statins, DEF or DEH), history of chronic diseases (HNT, NYHA functional class, and ischemic stroke). HR, hazard ratio; NYHA, New York Heart Association; HNT, hypertension. $\mathrm{ACEl}$, angiotensin converting enzyme inhibitors; ARB, angiotensin-receptor blocker; CCB, calcium channel blocker; DEF, diuretic except furosemide; $\mathrm{DEH}$, diuretic except hydrochlorothiazide.

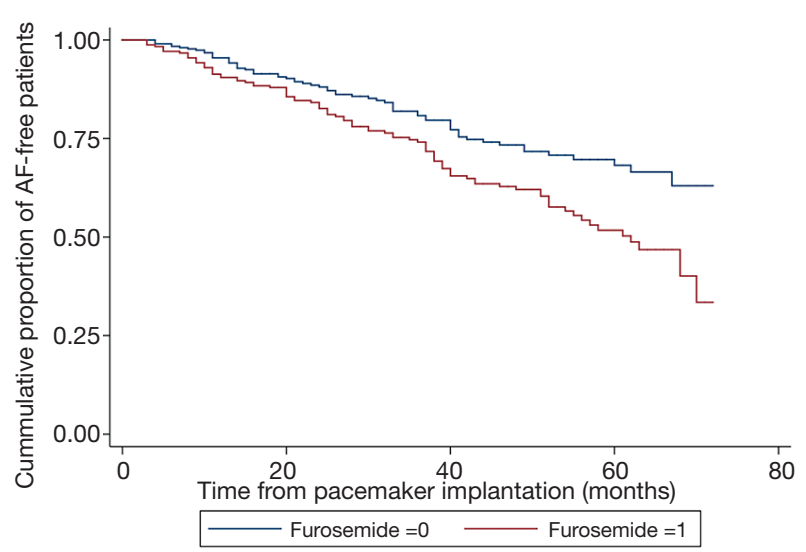

Figure 1 Kaplan-Meier curves showing cumulative 72 -month atrial fibrillation after pacemaker implantation in the furosemide group.

have been found to promote cardiac sympathetic meridian activation and increase the occurrence of arrhythmias. Right ventricular pacing and double-chamber pacing were capable of strongly increasing sympathetic tone (25), and abnormal excitement of autonomic nerves brought by artificial pacing may also lead to AF. Therefore, daily oral furosemide after artificial pacing may give rise to AF risk in the elderly via activating sympathetic nerves. Furosemide also plays a role in decreasing the level of magnesium in vivo. It is well established that renal magnesium reabsorption mainly takes place in the loop of Henle, and furosemide may induce substantial magnesium losses (26). The depletion of intracellular magnesium stores and hypomagnesaemia

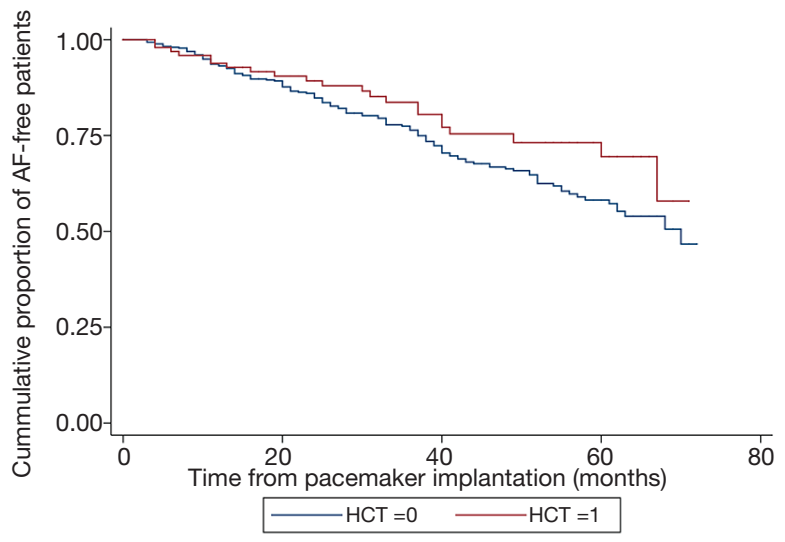

Figure 2 Kaplan-Meier curves showing cumulative 72-month atrial fibrillation after pacemaker implantation in the hydrochlorothiazide group. HCT, hydrochlorothiazide.

have been held responsible for a variety of cardiovascular abnormalities including $\mathrm{AF}$, and the mechanism might be underpinned by the enhanced electrical instability of the atrial and ventricular myocardium induced by intracellular magnesium depletion (27). In addition, the reduction of serum magnesium contributes to an inflammatory reaction in the heart, and the resultant interstitial fibrosis may serve as a substrate for development of AF. In summary, research suggests furosemide may induce pathologic remodeling of heart structure, activate sympathetic nerves, and reduce the level of serum magnesium, thereby potentially promoting the development of AF in elderly patients. These findings are consistent with the results of our study which show a higher risk of $\mathrm{AF}$ in the elderly population after pacemaker 
implantation relates to daily oral furosemide.

Hydrochlorothiazide may reduce heart pathologic structural remodeling via several mechanisms. Thiazidetype diuretics can reduce left ventricular hypertrophy and drawdown pathologic heart remodeling (13), and hydrochlorothiazide has been shown to decrease plasma proinflammatory cytokine levels and inhibit the TGF- $\beta$ / Smads signaling pathway in rats, thereby improving their cardiac function reducing cardiac fibrosis $(14,28)$. In addition, because TGF- $\beta$ acts downstream of Ang II, hydrochlorothiazide probably works through regulating the Ang II/TGF- $\beta$ cascade and research has shown that artificial pacing induces abnormal electrical excitability and hemodynamic changes, which may lead to increased serum Ang II levels. These findings suggest hydrochlorothiazide should be clinically preferred in elderly individuals as it decreases serum levels of Ang II and reduces the risk of AF after pacemaker implantation, and supports the results of our study which show hydrochlorothiazide provides a slight protective effect against $\mathrm{AF}$ after pacemaker implantation.

Daily oral furosemide increases the plasma level of renin/Ang II/aldosterone, activates sympathetic nerves, and reduces the level of serum magnesium, playing an aggregative role in the development of AF among elderly patients with permanent pacemakers. Therefore, maybe spironolactone, ACEI/ARB and magnesium could be used to reduce the side effect of furosemide.

On the contrary, daily oral hydrochlorothiazide reduces serum levels of angiotensin II, thereby inhibiting the TGF- $\beta /$ Smads signaling pathway to alleviate heart structural remodeling, and finally reduces the risk of $\mathrm{AF}$ in elderly patients after pacemaker implantation.

Multiple guidelines have advised that diuretics should be used for elderly patients suffering from HF or $\operatorname{HTN}(10,11)$. The 2018 ESC/ESH Guidelines for the management of arterial HTN recommended five major drug classes for the treatment of HTN: ACEIs, ARBs, $\beta$-blockers, CCBs, and diuretics, and diuretics are optimally advised especially in old and very old patients (29). While thiazides and related compounds, loop diuretics, and potassium-sparing diuretics are the main diuretics in use, which kind of diuretic to choose remains a controversial topic and should be determined according to the individual status of patients. Clinically, furosemide is widely used for HF, particularly in old individuals with fluid retention and renal failure. But for the old people with a permanent pacemaker, many drugs should be used to counteract the adverse effects.

Conversely, hydrochlorothiazide is recommended as a cornerstone therapy for antihypertension and small doses of it are often used in combination with other antihypertensive drugs like ACEI/ARB for elderly patients with HTN. Imparting a mild diuretic effect, potassium-sparing diuretics serve as adjuvant therapy for HF and HTN in clinical practice. For patients with gout or diabetes who are using hydrochlorothiazide, they could add the use of lowing uric acid drugs at the same time. Besides, another diuretics such as spirolactone would be a better choice for people in that situations. The results of our present study suggest that in elderly patients with a permanent pacemaker, hydrochlorothiazide may be preferable to furosemide by avoiding the risk of $\mathrm{AF}$ developing.

Overall, our results demonstrated that elderly individuals who followed daily oral furosemide after pacemaker implantation were associated with a raised risk of developing new $\mathrm{AF}$ in comparison to those not using it, which remained statistically significant after adjustment for related clinical covariates. In contrast, receiving hydrochlorothiazide therapy showed a slight protective effect against AF developing in elderly pacing patients. Our findings suggest that the daily use of hydrochlorothiazide is safer than oral furosemide in elderly patients with a permanent pacemaker for reducing the risk of subsequent AF. Our study may provide evidence for the clinical selection of diuretics for elderly pacing individuals.

\section{Limitation}

Our study has some limitations. Firstly, we only transferred the retrospective data from a single center based on case records, so the final sample size of the group is small. In addition, the definition of the onset time of AF may have a small range of bias because it is generally difficult to accurately define this by asking patients about their symptoms and medical records must be relied upon. Thirdly, the factors leading to the occurrence of AF are very complex. We have considered the correction factors as much as possible, but it is inevitable that there would still be some omissions. In addition, the pacing mode may also affect the incidence rate of subsequent $\mathrm{AF}$ after pacemaker implantation (30), and we did not categorize different pacing modes or conduct subgroup analyses because most of the included individuals received ventricular single chamber 
pacing (VVI mode).

\section{Conclusions}

Daily oral furosemide might enhance the risk of developing AF after pacemaker implantation in elderly patients. In contrast, hydrochlorothiazide has no detrimental effect and might be clinically preferred when needed.

\section{Acknowledgments}

Funding: This study was funded by the National Natural Science Foundation of China (No: 81974022), Shanghai Health Committee (201940206) and Xinhua Hospital Affiliated to Shanghai Jiao Tong University School of Medicine Chongming Branch (2019YA-02).

\section{Footnote}

Reporting Checklist: The authors have completed the STROBE reporting checklist. Available at http://dx.doi. org/10.21037/atm-21-1792

Data Sharing Statement: Available at http://dx.doi. org/10.21037/atm-21-1792

Conflicts of Interest: All authors have completed the ICMJE uniform disclosure form (available at http://dx.doi. org/10.21037/atm-21-1792). The authors have no conflicts of interest to declare.

Ethical Statement: The authors are accountable for all aspects of the work in ensuring that questions related to the accuracy or integrity of any part of the work are appropriately investigated and resolved. All data were anonymized. Individual clinical information could only be identified by using inpatient numbers derived from each participant's identification number before the research team received the data. Ethical approval was granted by the Medical Ethics Committee of the Xinhua Hospital Chongming Branch (CMEC-2020-KT-14), and our study obeyed the principles of the Declaration of Helsinki (as revised in 2013). The study was waived the need for informed consent due to the observational character of the study.

Open Access Statement: This is an Open Access article distributed in accordance with the Creative Commons
Attribution-NonCommercial-NoDerivs 4.0 International License (CC BY-NC-ND 4.0), which permits the noncommercial replication and distribution of the article with the strict proviso that no changes or edits are made and the original work is properly cited (including links to both the formal publication through the relevant DOI and the license). See: https://creativecommons.org/licenses/by-nc-nd/4.0/.

\section{References}

1. Gregoratos G, Abrams J, Epstein AE, et al. ACC/AHA/ NASPE 2002 guideline update for implantation of cardiac pacemakers and antiarrhythmia devices: summary article. A report of the American College of Cardiology/American Heart Association Task Force on Practice Guidelines (ACC/AHA/NASPE Committee to Update the 1998 Pacemaker Guidelines). J Cardiovasc Electrophysiol 2002;13:1183-99.

2. Nikolaidou T, Fox DJ, Brown BD. Bradycardia pacing. Medicine 2018;46:646-51.

3. Kirchhof P, Benussi S, Kotecha D, et al. 2016 ESC Guidelines for the Management of Atrial Fibrillation Developed in Collaboration With EACTS. Rev Esp Cardiol (Engl Ed) 2017;70:50.

4. Sweeney MO, Hellkamp AS, Ellenbogen KA, et al. Adverse effect of ventricular pacing on heart failure and atrial fibrillation among patients with normal baseline QRS duration in a clinical trial of pacemaker therapy for sinus node dysfunction. Circulation 2003;107:2932-7.

5. Connolly SJ, Kerr CR, Gent M, et al. Effects of physiologic pacing versus ventricular pacing on the risk of stroke and death due to cardiovascular causes. Canadian Trial of Physiologic Pacing Investigators. N Engl J Med 2000;342:1385-91.

6. Opacic D, van Bragt KA, Nasrallah HM, et al. Atrial metabolism and tissue perfusion as determinants of electrical and structural remodelling in atrial fibrillation. Cardiovasc Res 2016;109:527-41.

7. Korantzopoulos P, Letsas KP, Tse G, et al. Inflammation and atrial fibrillation: A comprehensive review. J Arrhythm 2018;34:394-401.

8. Jalife J, Kaur K. Atrial remodeling, fibrosis, and atrial fibrillation. Trends Cardiovasc Med 2015;25:475-84.

9. Mayyas F, Alzoubi KH, Van Wagoner DR. Impact of aldosterone antagonists on the substrate for atrial fibrillation: Aldosterone promotes oxidative stress and atrial structural/electrical remodeling. Int J Cardiol 2013;168:5135-42. 
10. Yancy CW, Jessup M, Bozkurt B, et al. 2013 ACCF/AHA Guideline for the Management of Heart Failure. A Report of the American College of Cardiology Foundation/ American Heart Association Task Force on Practice Guidelines. J Am Coll Cardiol 2013;62:e147-239.

11. Ponikowski P, Voors AA, Anker SD, et al. 2016 ESC Guidelines for the diagnosis and treatment of acute and chronic heart failure. Eur J Heart Fail 2016;18:891-975.

12. Goldberger JJ, Johnson NP, Gidea C. Significance of Asymptomatic Bradycardia for Subsequent Pacemaker Implantation and Mortality in Patients $>60$ Years of Age. Am J Cardiol 2011;108:857-61.

13. Roush GC, Abdelfattah R, Song S, et al. Hydrochlorothiazide and alternative diuretics versus renin-angiotensin system inhibitors for the regression of left ventricular hypertrophy: a head-to-head meta-analysis. J Hypertens 2018;36:1247-55.

14. Lantis AC, Atkins CE, DeFrancesco TC, et al. Effects of furosemide and the combination of furosemide and the labeled dosage of pimobendan on the circulating reninangiotensin-aldosterone system in clinically normal dogs. Am J Vet Res 2011;72:1646-51.

15. Dobaczewski $M$, Chen W, Frangogiannis NG.

Transforming growth factor (TGF)- signaling in cardiac remodeling. J Mol Cell Cardiol 2011;51:600-6.

16. Boriani G, Biffi M, Martignani C, et al.

Electrocardiographic remodeling during cardiac resynchronization therapy. Int J Cardiol 2006;108:165-70.

17. Chen XL, Ren XJ, Liang Z, et al. Analyses of risk factors and prognosis for new-onset atrial fibrillation in elderly patients after dual-chamber pacemaker implantation. J Geriatr Cardiol 2018;15:628-33.

18. Karnik AA, Gopal DM, Ko D, et al. Epidemiology of Atrial Fibrillation and Heart Failure: A Growing and Important Problem. Cardiol Clin 2019;37:119-29.

19. Fauchier L, de Groote P. Atrial fibrillation and reninangiotensin-aldosterone system: believe it or not. Europace 2011;13:297-8.

Cite this article as: Lin DW, Jiang F, Wu C, Li YG, Zhang X, Wang YS. Association of furosemide or hydrochlorothiazide use with risk of atrial fibrillation post pacemaker implantation among elderly patients. Ann Transl Med 2021;9(10):855. doi: 10.21037/atm-21-1792
20. Eshaghian S, Horwich TB, Fonarow GC. Relation of Loop Diuretic Dose to Mortality in Advanced Heart Failure. Am J Cardiol 2006;97:1759-64.

21. Lijnen P, Petrov V. Induction of Cardiac Fibrosis by Aldosterone. J Mol Cell Cardiol 2000;32:865-79.

22. Qu YC, Du YM, Wu SL, et al. Activated nuclear factorkappaB and increased tumor necrosis factor-alpha in atrial tissue of atrial fibrillation. Scand Cardiovasc J 2009;43:292-7.

23. Corsini WA, Hook JB, Bailie MD. Control of renin secretion in the dog. Effects of furosemide on the vascular and macula densa receptors. Circ Res 1975;37:464-70.

24. Martens P, Verbrugge FH, Nijst P, et al. Changes in Loop Diuretic Dose and Outcome After Cardiac Resynchronization Therapy in Patients With Heart Failure and Reduced Left Ventricular Ejection Fractions. Am J Cardiol 2017;120:267-73.

25. Shen MJ, Choi EK, Tan AY, et al. Patterns of baseline autonomic nerve activity and the development of pacinginduced sustained atrial fibrillation. Heart Rhythm 2011;8:583-9.

26. Reinhart RA. Magnesium Metabolism: A Review With Special Reference to the Relationship Between Intracellular Content and Serum Levels. Arch Intern Med 1988;148:2415-20.

27. Weber KT. Furosemide in the long-term management of heart failure. The good, the bad, and the uncertain. J Am Coll Cardiol 2004;44:1308-10.

28. Leask A. TGF $\beta$, cardiac fibroblasts, and the fibrotic response. Cardiovasc Res 2007;74:207-12.

29. Williams B, Mancia G, Spiering W, et al. (2018 ESC/ESH Guidelines for the management of arterial hypertension). Kardiol Pol 2019;77:71-159.

30. Zhang L, Jiang H, Wang W, et al. Interatrial septum versus right atrial appendage pacing for prevention of atrial fibrillation. Herz 2018;43:438-46.

(English Language Editor: B. Draper) 